\title{
Administration of leukemia inhibitory factor increases Opalin and myelin oligodendrocyte glycoprotein expression in the cerebral cortex in a cuprizone-induced model of demyelination
}

\author{
Farhad Mashayekhi, Sara Pishgah Hadiyan, Zivar Salehi \\ Department of Biology, Faculty of Sciences, University of Guilan, Rasht, Iran
}

\begin{abstract}
Multiple sclerosis (MS) lesions are characterized by inflammatory demyelination and reactive gliosis, and although remyelination occurs in some lesions it is limited and incomplete. Leukemia inhibitory factor (LIF) is an important cytokine that stimulates oligodendrocyte proliferation and survival in vitro. Opalin is a unique molecular marker for mature oligodendrocytes. The aim of this study was to demonstrate the role of LIF on Opalin and myelin oligodendrocyte glycoprotein (MOG) expression in the cerebral cortex of cuprizone-induced MS mice. The mice were treated with cuprizone for five weeks in order to induce MS. The mice were then divided into 3 groups. The first group was injected intraperitoneally (IP) with LIF for six weeks in the amount of $30 \mu \mathrm{g} / \mathrm{kg}$ bw per day. The second group (SHAM) was injected IP with normal saline and the third group was left without injection as a control. After six weeks the mice were killed, the cerebral cortex was harvested, and the expression of MOG and Opalin was studied. Using western blotting we found that LIF increases Opalin and MOG expression in the cerebral cortex extracts as compared to SHAM and control groups. However, no significant difference in the Opalin and MOG expression was seen between SHAM and control groups. It is concluded that LIF may have an important role in the process of remyelination by increasing Opalin expression and MOG expression.
\end{abstract}

Key words: leukemia inhibitory factor, Opalin, myelin oligodendrocyte glycoprotein, cuprizone, remyelination.

\section{Introduction}

Multiple sclerosis (MS) is a chronic demyelinating disease of the central nervous system (CNS). Approximately 2.5 million people currently live with MS globally. Susceptibility to MS varies in different ethnic groups, with people of Scottish origin being more likely to develop the disease [5]. Family members of MS patients have a higher risk of developing the disease than individuals without a family history [1].

Multiple sclerosis lesions are characterized by inflammatory demyelination and reactive gliosis, and although remyelination occurs in some lesions it is limited and incomplete. Possible reasons for this abortive remyelination include astrogliosis and accumulation of extracellular matrix, lack of myelinating 
cells or an unfavorable combination of growth factors. Growth factor expression could participate in the repair process of this demyelinating disease by modulating the activity of microglia/macrophages in an autocrine fashion, by inducing the expression of other factors that can affect myelin regeneration or degeneration, and also by directly stimulating the localized proliferation and/or regeneration of oligodendrocytes within lesion areas [21]. It has been shown that one of the identified proteins, transmembrane protein 10 (Tmem10; also known as Opalin or oligodendrocyte paranodal loop protein), whose expression is specific to the brain, predominantly localized in many white matter regions and was upregulated during the postnatal developmental stages [2]. It was shown that Opalin protein is specifically expressed by myelinating oligodendrocytes, suggesting that it plays a unique role in mammalian myelin [11]. In the CNS the main proteins of myelin are proteolipid protein (PLP), myelin basic protein (MBP) and myelin oligodendrocyte glycoprotein (MOG). Myelin oligodendrocyte glycoprotein is a minor component of the myelin sheath, but it is an important autoantigen linked to the pathogenesis of MS. It has been shown that MOG is a potent encephalitogen that triggers strong $T$ cell and $B$ cell responses. It has been recently shown that MOG antibodies could be associated with a broad spectrum of acquired human CNS demyelinating diseases [20].

Cytokines and growth factor play important roles in brain development and function [14,15]. Growth factor expression could participate in the repair process of demyelinating disease by modulating the activity of microglia/macrophages, inducing the expression of other factors that can affect myelin regeneration or degeneration, and also by directly stimulating the localized proliferation and/or regeneration of oligodendrocytes within lesion areas [21]. Among growth factors, leukemia inhibitory factor (LIF) is a cytokine that has been demonstrated to play an important role in neural cell survival, including cholinergic neurons. It has been demonstrated that LIF limits autoimmune demyelination and oligodendrocyte loss in a murine model of MS [7]. Leukemia inhibitory factor promotes the differentiation of either neurons or astrocytes depending on the culture conditions [19]. Increased LIF concentration in the cerebrospinal fluid (CSF) of patients with MS has been demonstrated [16]. It has been suggested that LIF is a potential therapeutic candidate for MS [23].
Leukemia inhibitory factor binds to a heterodimeric membrane receptor complex consisting of LIF receptor (LIFR) and glycoprotein 130 (gp120) [25]. Leukemia inhibitory factor receptor has been shown to be expressed in the dorsal ventricular zone and subventricular zone of the cerebral cortex [4]. Co-operative expression of LIF and LIFR in the brain of patients with neurodegenerative disease including Parkinson's disease (PD) and Alzheimer's disease (AD) may indicate a role for LIF in neuronal damage or repair in these diseases [24]. It was shown that LIF delivery to oligodendrocyte progenitor cells (OPCs) stimulates their proliferation through the activation of gp130 receptor signaling within these cells, and it has been suggested that LIF has both reparative and protective activities that make it a promising potential therapy for CNS demyelinating disorders and injuries [9]. It has been shown that LIF is a neurotrophic cytokine and plays an important role in neural cell survival and myelin formation in vitro [13]. It has been shown that suppressing expression of CNTF, which is an LIF family, plays a key role in the remyelination process in cuprizone-induced demyelinated mouse [26]. Addition of LIF neutralizing antibodies inhibited oligodendrocyte differentiation, indicating a crucial role of TNFR2-induced astrocyte derived LIF for oligodendrocyte maturation [10].

As Opalin is a unique molecular marker for mature oligodendrocytes and MOG is a component of the myelin sheath which is an important autoantigen linked to the pathogenesis of MS $[11,20]$, we aimed to examine the in vivo effects of LIF on the remyelination process in the cerebral cortex of the cuprizone-induced MS mouse.

\section{Material and methods \\ Animals}

Balb/c mice were purchased from Pasteur Institute, Tehran, Iran and maintained on a 12-12 light: dark cycle beginning at $8.00 \mathrm{am}$. They were kept at a constant temperature in mouse boxes with unrestricted access to laboratory food and water. The colony was maintained through random pair mating. Timed mating was ca rried out by placing a male and female together and checking for the presence of a vaginal plug. The presence of a vaginal plug was taken as gestational day zero (EO) and the day of birth was designated postnatal day 0 (PO). 


\section{Induction of demyelination and treatment with leukemia inhibitory factor}

Demyelination was induced by feeding 8-10-weekold mice a diet containing $0.2 \%$ cuprizone (bis-cyclohexanone oxaldihydrazone, Sigma-Aldrich Inc.) mixed into ground standard rodent chow.

The cuprizone diet was administered for 5 weeks for demyelination. The control group received breeder chow without the cuprizone admixture. Animals were then put on standard rodent chow without cuprizone to induce remyelination. The mice were then divided into three groups. The first group was injected intraperitoneally (IP) with LIF for 6 weeks in the amount of $30 \mu \mathrm{g} / \mathrm{kg}$ bw per day. The second group (SHAM) was injected with normal saline and the third group was left without injection as the control group. After four weeks the cerebral cortex was harvested after euthanasia by intraperitoneal injection of an overdose of anesthetic (sodium pentobarbitone) and the cerebral cortex was removed and processed as described. In total, 42 animals were used in this study ( $n=14$ for each group).

\section{Cell extract}

Fresh tissue samples (10 mg each) were chopped into tiny pieces and suspended in $0.5 \mathrm{ml}$ of protein lysis buffer [150 mM NaCl, 1.0\% NP40, 20 mM Tris (pH 7.5), $5 \mathrm{mM}$ EDTA, and Complete Mini protease inhibitor cocktail (Roche Diagnostics Ltd., West Sussex, UK)] and then mechanically homogenized by sonication. After centrifugation, the protein extracts were recovered and stored at $-70^{\circ} \mathrm{C}$ until they were used.

\section{Total protein concentration and western blotting}

The total protein concentration in the cerebral cortex extracts was determined by the Bio-Rad protein assay based on the Bradford dye procedure. For western blot, protein extracts (50 $\mu \mathrm{g} /$ lane) were separated on $10 \%$ SDS-polyacrylamide gel and transferred to a polyvinylidene difluoride membrane (Bio-Rad Laboratories Ltd. Hertfordshire, UK). The membranes were blocked with phosphate-buffered saline (PBS) containing 0.05\% Tween 20 and $5 \%$ dry milk and probed either with polyclonal anti-Opalin antibody (Santa Cruz Biotechnology; sc-135362) (1 : 1000 dilution), polyclonal anti-MOG antibody or a mouse monoclonal anti- $\beta$-tubulin antibody (as a loading control) (Abcam plc, Cambridge, UK) (1 : 10,000 dilution) and then treated with the appropriate horseradish peroxidase-conjugated secondary antibodies. Immunoreactive protein was visualized using the Enhanced Chemiluminescence western blotting detection system (Amersham Pharmacia Biotech, Piscataway, NJ). Densitometric analysis was performed by scanning immunoblots and quantitating protein bands using an image analyzer (Metaview Software).

\section{Statistical analysis}

All data presented are expressed as mean \pm standard error of the mean (SEM). Statistical analysis was performed using the one-way ANOVA to test for differences among the groups, and only values with $p \leq 0.05$ were considered significant.

\section{Results}

\section{Total protein concentration}

The total protein concentration in the cerebral cortex extracts from LIF injected, SHAM and control groups was determined by the Bio-Rad protein assay based on the Bradford dye mixture. The total protein contents of LIF injected, SHAM and control were $1.11 \pm 0.12,1.07 \pm 0.18$ and $1.05 \pm 0.18(\mathrm{~g} / \mathrm{l})$, respectively. No significant increase in the total protein concentration was seen in the LIF-injected brain samples compared with those from the SHAM and control groups $(p>0.05)$.

\section{Analysis of Opalin and myelin oligodendrocyte glycoprotein expression by western blotting}

Western blot analysis was performed to quantitatively evaluate Opalin and MOG expression in the cerebral cortical extracts. A western blot analysis using anti-Opalin and MOG antibodies as a probe confirmed the presence of Opalin and MOG in all the extracts (Fig. 1A and Fig. 2A). An image analyzer was used to determine the intensities of the band in the respective lanes. Quantification of the western blot bands from repeated experiments $(n=14)$ showed that the amount of Opalin and MOG was significantly increased in the LIF-injected cerebral cortical extracts when compared with SHAM and control groups $(p<0.0001)$ (Fig. 1B and Fig. 2B). 
A
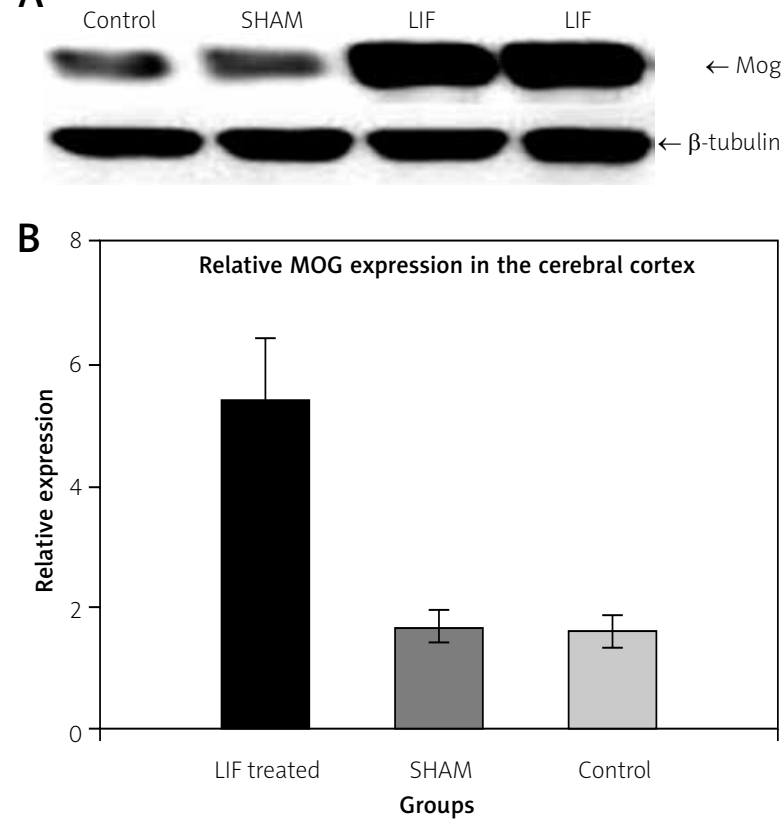

Fig. 1. (A) Myelin oligodendrocyte glycoprotein (MOG) expression in the cerebral cortex extracts from leukemia inhibitory factor (LIF) treated, SHAM and control groups. $\beta$-tubulin (50 kDa) expression was determined as a protein loading control. (B) Signal intensities from MOG expression in the LIF treated, SHAM and control cerebral cortex immunoblotting experiments were determined by densitometric analysis. In each of the experimental groups the number of animals investigated was 14 . A significant increase in MOG expression was seen in the LIF-injected group when compared with SHAM and control groups $(p<0.001)$. No significant differences were seen between the SHAM and control group $(p=0.42)$.

\section{Discussion}

Acute demyelination of the CNS in MS is initially followed by a process of remyelination [3]. This repair process is guaranteed by the extensive proliferation of oligodendrocyte progenitor cells (OPCs) in response to demyelination. It has been demonstrated that growth factor expression could be important in the repair process of this demyelinating disease by modulating the activity of microglia/macrophages by inducing the expression of other factors that can affect myelin regeneration and also by directly stimulating the localized proliferation and/or regeneration of oligodendrocytes within lesion areas.
A
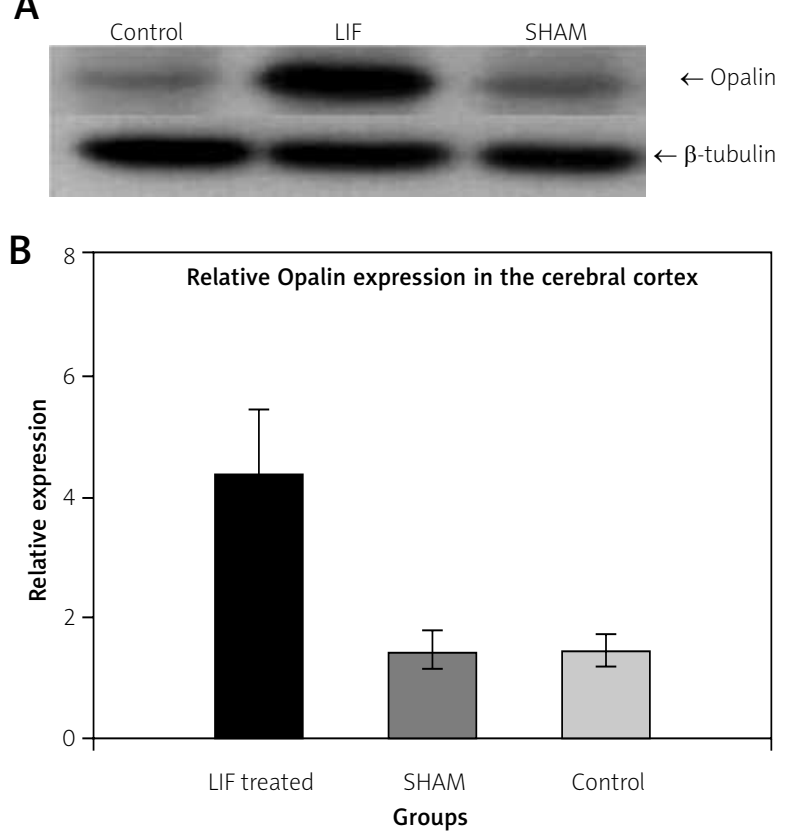

Fig. 2. (A) Opalin expression in the cerebral cortex extracts from leukemia inhibitory factor (LIF) treated, SHAM and control groups. $\beta$-tubulin (50 kDa) expression was determined as a protein loading control. (B) Signal intensities from Opalin expression in the LIF treated, SHAM and control cerebral cortex immunoblotting experiments was determined by densitometric analysis. In each of the experimental groups the number of animals investigated was 14. A significant increase in Opalin expression was seen in the LIF-injected group when compared with SHAM and control groups $(p<0.001)$. No significant difference was seen between SHAM and control groups $(p=0.82)$.

Among growth factors, LIF has been shown to play an important role in neural cell survival [8]. Leukemia inhibitory factor has been demonstrated to limit autoimmune demyelination and oligodendrocyte loss in a murine model of MS [6]. Spinal cord precursors also synthesize LIF, and anti-LIFR $\beta$ antibodies completely blocked the generation of neurons. Leukemia inhibitory factor plays an important role in generation, maturation and survival of oligodendrocytes [17]. Data supporting a neuroprotective role of LIF have been obtained in MS, where LIF has been demonstrated to be produced by myelin-reactive $\mathrm{T}$ cells isolated from MS patients and protect 
against tumor necrosis factor- $\alpha$-induced oligodendrocyte apoptosis [27].

We investigated LIF as it is one of the most important growth factors involved in the survival of oligodendrocytes [12]. Leukemia inhibitory factor contributes to differentiation of the glial cell lineage [18].

The role of ciliary neurotrophic factor (CNTF) in the process of remyelination has been demonstrated [22]. Here we show that daily administration of LIF significantly increases MOG and Opalin expression in the cuprizone-induced mouse cerebral cortex. As Opalin is a unique molecular marker for mature oligodendrocytes, the increased Opalin expression in the cerebral cortex of LIF injected mouse may be due to increased differentiation of oligodendrocyte progenitor cells into mature oligodendrocytes. We have also shown that LIF increases MOG expression in the cerebral cortex, which indicates the role of LIF in myelin formation. The results of this study could have been anticipated with some certainty given the findings of other studies that have investigated the role of LIF in MS.

It is thus concluded that LIF may have an important role in the process of remyelination by increasing either Opalin expression and oligodendrocyte differentiation or MOG expression. It is also suggested that LIF may have an important role in the process of remyelination by increasing MOG expression in the cerebral cortex.

\section{Acknowledgements}

This study was supported by the University of Guilan.

\section{Disclosure}

Authors report no conflict of interest.

\section{References}

1. Al Jumah M, Kojan S, Al Khathaami A, Al Abdulkaream I, Al Blawi M, Jawhary A. Familial multiple sclerosis: does consanguinity have a role? Mult Scler 2011; 17: 487-489.

2. Aruga J, Yoshikawa F, Nozaki Y, Sakaki Y, Toyoda A, Furuichi T. An oligodendrocyte enhancer in a phylogenetically conserved intron region of the mammalian myelin gene Opalin. J Neurochem 2007; 102: 1533-1547.

3. Barnett $M H$, Sutton I. The pathology of multiple sclerosis: a paradigm shift. Curr Opin Neurol 2006; 9: 242-247.

4. Bauer S, Rasika S, Han J, Mauduit C, Raccurt M, Morel G, Jourdan F, Benahmed M, Moyse E, Patterson PH. Leukemia inhibitory factor is a key signal for injury-induced neurogenesis in the adult mouse olfactory epithelium. J Neurosci 2003; 23: 17921803.

5. Bayes HK, Weir CJ, O'Leary C. Timing of birth and risk of multiple sclerosis in the Scottish population. Eur Neurol 2010; 63: 36-40.

6. Butzkueven H, Emery B, Cipriani T, Marriott MP, Kilpatrick TJ. Endogenous leukemia inhibitory factor production limits autoimmune demyelination and oligodendrocyte loss. Glia 2006; 53: 696-703.

7. Butzkueven H, Zhang JG, Soilu-Hanninen M, Hochrein H, Chionh F, Shipham KA, Emery B, Turnley AM, Petratos S, Ernst M, Bartlett PF, Kilpatrick TJ. LIF receptor signaling limits immune-mediated demyelination by enhancing oligodendrocyte survival. Nat Med 2002; 8: 613-619.

8. Cheema SS, Arumugam D, Murray SS, Bartlett PF. Leukemia inhibitory factor maintains choline acetyltransferase expression in vivo. Neuroreport 1998; 9: 363-366.

9. Deverman BE, Patterson PH. Exogenous leukemia inhibitory factor stimulates oligodendrocyte progenitor cell proliferation and enhances hippocampal remyelination. J Neurosci 2012; 32: 2100-2109.

10. Fischer R, Wajant H, Kontermann R, Pfizenmaier K, Maier O. Astrocyte-specific activation of TNFR2 promotes oligodendrocyte maturation by secretion of leukemia inhibitory factor. Glia 2014; 62: 272-283.

11. Golan N, Adamsky K, Kartvelishvily E, Brockschnieder D, Möbius W, Spiegel I, Roth AD, Thomson CE, Rechavi G, Peles E. Identification of Tmem10/Opalin as an oligodendrocyte enriched gene using expression profiling combined with genetic cell ablation. Glia 2008; 56: 1176-1186.

12. Ishibashi T, Lee PR, Baba H, Fields RD. Leukemia inhibitory factor regulates the timing of oligodendrocyte development and myelination in the postnatal optic nerve. J Neurosci Res 2009; 87: 3343-3355.

13. Marriott MP, Emery B, Cate HS, Binder MD, Kemper D, Wu Q, Kolbe S, Gordon IR, Wang H, Egan G, Murray S, Butzkueven H, Kilpatrick TJ. Leukemia inhibitory factor signaling modulates both central nervous system demyelination and myelin repair. Glia 2008; 56: 686-698.

14. Mashayekhi F, Gholizadeh L. Administration of anti-c-kit antibody into the cerebrospinal fluid leads to increased cell death in the developing cerebral cortex. Saudi J Biol Sci 2011; 18: 261-266.

15. Mashayekhi F, Sadeghi M, Rajaei F. Induction of perlecan expression and neural cell proliferation by FGF-2 in the developing cerebral cortex: an in vivo study. J Mol Neurosci 2011; 45: 87-93.

16. Mashayekhi F, Salehi Z. Expression of leukemia inhibitory factor in the cerebrospinal fluid of patients with multiple sclerosis. J Clin Neurosci 2011; 18: 951-954.

17. Mayer M, Bhakoo K, Noble M. Ciliary neurotrophic factor and leukemia inhibitory factor promote the generation, maturation and survival of oligodendrocytes in vitro. Development 1994; 120: 143-153.

18. Nakagaito Y, Yoshida T, Satoh M, Takeuchi M. Effects of leukemia inhibitory factor on the differentiation of astrocyte progenitor cells from embryonic mouse cerebral hemispheres. Brain Res Dev Brain Res 1995; 87: 220-223. 
19. Nakanishi M, Niidome T, Matsuda S, Akaike A, Kihara T, Sugimoto H. Microglia-derived interleukin-6 and leukaemia inhibitory factor promote astrocytic differentiation of neural stem/progenitor cells. Eur J Neurosci 2007; 25: 649-658.

20. Reindl M, Di Pauli F, Rostásy K, Berger T. The spectrum of MOG autoantibody-associated demyelinating diseases. Nat Rev Neurol 2013; 9: 455-461.

21. Rosenberg SS, Ng BK, Chan JR. The quest for remyelination: a new role for neurotrophins and their receptors. Brain Pathol 2006; 16: 288-294.

22. Salehi Z, Hadiyan SP, Navidi R. Ciliary neurotrophic factor role in myelin oligodendrocyte glycoprotein expression in Cuprizoneinduced multiple sclerosis mice. Cell Mol Neurobiol 2013; 33: 531-535.

23. Slaets H, Hendriks JJ, Stinissen P, Kilpatrick TJ, Hellings N. Therapeutic potential of LIF in multiple sclerosis. Trends Mol Med 2010; 16: 493-500

24. Soilu-Hänninen M, Broberg E, Röyttä M, Mattila P, Rinne J, Hukkanen V. Expression of LIF and LIF receptor beta in Alzheimer's and Parkinson's diseases. Acta Neurol Scand 2010; 121: 44-50.

25. Takahashi Y, Carpino N, Cross JC, Torres M, Parganas E, Ihle JN. SOCS3: an essential regulator of LIF receptor signaling in trophoblast giant cell differentiation. EMBO I 2003; 22: 372-384.

26. Tanaka T, Murakami K, Bando Y, Yoshida S. Minocycline reduces remyelination by suppressing ciliary neurotrophic factor expres sion after cuprizone-induced demyelination. J Neurochem 2013; 127: 259-270

27. Vanderlocht J, Hellings N, Hendriks JJ, Vandenabeele F, Moreels M, Buntinx M, Hoekstra D, Antel JP, Stinissen P. Leukemia inhibitory factor is produced by myelin-reactive T cells from multiple sclerosis patients and protects against tumor necrosis factor-alpha-induced oligodendrocyte apoptosis. J Neurosci Res 2006; 83: 763-774. 